\title{
Proyecto Dorothy: creación y puesta en marcha del "Clúster Logística Urbana de la Comunitat Valenciana"
}

\author{
Oscar Colomer Font \\ Investigador contratado, Universitat Politècnica de València, España \\ José Vicente Colomer Ferrándiz \\ Catedrático de Transportes, Universitat Politècnica de València, España \\ Mauro Fiore \\ Gerente, MOVUS SL (Movilidad Urbana Sostenible), España
}

\section{RESUMEN}

En julio de 2013 comenzó el proyecto DOROTHY "Development Of RegiOnal clusTers for researcH and implementation environmental friendlY urban logistics" (www.clusterdorothy.com), que forma parte del 7th Framework Programme of the EU. Dicho proyecto está centrado en la logística urbana. Su fecha de finalización, 30 de Junio de 2016, coincide prácticamente con la celebración del Congreso de Ingeniería de Transporte CIT 2016.

Uno de los objetivos principales del Proyecto Dorothy es la creación de clusters, a nivel regional que aborden temas relacionados con la logística urbana. Uno de estos clústers se ha desarrollado en la Región de Valencia.

La creación de un clúster en logística urbana, y su puesta en marcha, sin disponer de fondos económicos para su dinamización inicial, es un proceso complejo que requiere abordar diversos pasos con continuidad y llevar a cabo distintas gestiones. Al menos este ha sido el caso de la creación del clúster de la Región de Valencia.

Entendemos que explicar este proceso con cierto detalle puede resultar de interés para futuras creaciones de asociaciones o clústers relacionados con la logística urbana o con el transporte en sus diversos aspectos.

La presente ponencia recoge las distintas acciones que han sido necesarias para la creación del clúster, así como las primeras actuaciones del mismo que ponen de manifiesto el dinamismo inicial de esta iniciativa. 


\section{1.- INTRODUCCIÓN}

La distribución urbana de mercancías (logística urbana) es una actividad muy importante dentro de las ciudades tanto desde el punto de vista social y económico. Por una parte garantiza los suministros necesarios para la vida en la ciudad, y por otra incide en diversos aspectos relacionados con el tráfico y el medioambiente.

En la logística urbana (LU) intervienen distintos actores con intereses, en ocasiones, contrapuestos: transportistas, comerciantes, ciudadanos y administraciones municipales y regionales. Para poder optimizar esta actividad es necesaria la participación de todos los afectados.

En este sentido, el proyecto DOROTHY "Development Of RegiOnal clusTers for researcH and implementation environmental friendlY urban logistics" (www.clusterdorothy.com), forma parte del 7th Framework Programme of the EU en la sección "Transnational cooperation between regional research-driven clusters" ${ }^{1}$, que se inició en Junio de 2013 y que termina a final de Junio de 2016 plantea como uno de sus objetivos principales la creación de clusters, a nivel regional que se encarguen de los temas relacionados con la logística urbana.

Las regiones que participan en el proyecto son: región de Valencia (España) con cinco partners, región de Toscana(Italia) con siete partners, región Lisboa y Tajo (Portugal) con siete partners y región de Oltenia con seis partners. Del total de 25 miembros, cuatro son Universidades o Institutos de Investigación (uno por región), ocho son empresas privadas y los otros trece son entidades públicas municipales o regionales.

La presente ponencia recoge los pasos dados para la creación del clúster en logística urbana de la región de Valencia así como las primeras acciones que está llevando a cabo. Se confirma así el logro de uno de los objetivos fundamentales del proyecto Dorothy.

\section{2.- ACTUACIONES PREVIAS}

En la fase inicial del proyecto Dorothy, en el WP1, se analizó con detalle la estructura logística de la Región de Valencia en lo que se refiere a la logística urbana, llevando a cabo múltiples entrevistas personales con empresas, municipios, asociaciones de logística, proyectos similares existentes, etc. Este análisis permitió, por una parte,

\footnotetext{
${ }^{1}$ Eva María Pino, Davinia San Nicolás, Isabel Espinós: "Dorothy project: urban logistics organization in Valencian Community". XI Congreso de Ingeniería del Transporte (CIT 2014). Procedia - Social and Behavioral Sciences 160 ( 2014 ) 420 - 429. Elsevier 2014
} 
contactar con los principales actores que intervienen en la LU, y por otro, conocer tanto sus necesidades como sus puntos fuertes y los aspectos en los que su aportación podía resultar de mayor interés.

En las entrevistas que se realizaron, se introdujo el posible interés en la creación de un clúster de logística urbana así como las características que debía tener. Se constató una respuesta muy positiva por parte de algunos agentes entrevistados $\mathrm{y}$, en todos los casos, un cierto interés en participar o al menos estar informados de las actuaciones que lleve a cabo el posible clúster.

Debe tenerse en cuenta que el planteamiento, en la región de Valencia, se realiza en un momento de crisis (2013-2014), a un colectivo con una gran atomización, sin referentes organizativos previos en la materia específica y en un entorno poco habituado a los clústers. Por ello, el esfuerzo inicial previo tuvo que ser intenso e importante.

Con toda la información disponible, y con el fin de iniciar la constitución del clúster sobre logística urbana, se organizó, el 22 de Mayo de 2014, diez meses después del comienzo del proyecto Dorothy, una reunión en la Cámara de Comercio de Valencia con el tema: "El nuevo clúster de lo logística urbana de la Comunidad Valenciana". En este primer acto se presentó el proyecto Dorothy, se trató sobre la necesidad de realizar un Joint Action Plan (JAP), se puso de manifiesto la necesidad de una logística urbana sostenible dentro de una movilidad sostenible y se expusieron razones acerca de la conveniencia de crear un clúster de LU en la Comunidad Valenciana. El acto finalizó con una Mesa Redonda en la que participaron diversos asistentes.

Esta primera reunión, con alrededor de un centenar de asistentes, fue el punto de partida para la creación del clúster. La principal conclusión que se obtuvo fue que existía interés en el clúster y que el proyecto de creación del mismo resultaba viable.

La difusión del proyecto Dorothy así como el anuncio de la creación del clúster de logística urbana se continuó y se aprovecharon las oportunidades existentes para participar en distintos actos que diesen soporte y animasen la iniciativa del clúster.

En Septiembre de 2014, dentro de la Jornada Infoday Proebike, que se celebró en el Museo de Ciudad de Valencia, el 17 de septiembre de 2014, se incluyó una ponencia con el título "Dorothy: El nuevo Cluster de la logística urbana de la Comunidad Valenciana" que se refería, textualmente a: "Presentación del Cluster Dorothy cuya misión es mejorar el proceso de distribución de bienes urbanos mediante la reducción del número de vehículos y la mejora de las normas ambientales". El autor y ponente fue Mauro Fiore de la empresa MOVUS y que es el CCP ("Cluster Contact Point") de la región de Valencia.11 


\section{3.- LA CREACIÓN DEL CLÚSTER}

Tras la reunión de la Cámara de Comercio, y la posterior difusión del proyecto del clúster, se llevaron a cabo por parte de los partners del proyecto Dorothy de la región de Valencia, contactos directos con las empresas y entidades que mostraron mayor interés y para el lanzamiento del clúster se celebró una reunión -ya orientada directamente a la creación del clúster- en IVACE ${ }^{2}$.

Dicha reunión tuvo lugar el 16 de Octubre de 2014 y a la misma asistieron del orden de un centenar de personas pertenecientes a diversas empresas y entidades con interés en la logística urbana. El tema central de la reunión fue presentar propuestas para la organización del clúster así como ideas para abordar un borrador sobre los estatutos del clúster.

Especialmente importante, para la creación de estos entes, es atender a los aspectos de la figura legal que se adopta. Precisamente en este sentido se vio que debía partirse de una figura que, por una parte tuviese entidad jurídica, y por otra fuese suficientemente flexible ya que en los inicios el nivel de compromiso de los socios, en el plano jurídico $y$, sobre todo, en el plan o económico, no era muy alto. Debe tenerse en cuenta que la situación de crisis del sector, así como la características del mismo, hacía que presentase reticencias muy grandes a la adquisición de obligaciones. A esto se unió (y se une) que la situación económica del Gobierno Regional le impide adquirir compromisos de apoyo más allá de programas de la UE que, para este tema y en estos momentos no atienden la creación de clústers.

Se realizó un análisis de las distintas figuras posibles y se concluyó que para el caso de la región de Valencia la figura que mejor se adaptaba es la de "Asociación sin ánimo de lucro" al amparo de la Ley Orgánica 1/2002 de 22 de marzo reguladora del Derecho de Asociación.

En la reunión se acordó, en primer lugar, que todos los interesados en pertenecer al clúster debían solicitarlo y nombrar a un representante, en segundo lugar que se enviaría un borrador de estatutos que sirviera de base para el funcionamiento del clúster, y, por último, se estableció una fecha para la constitución del clúster, así como el nombramiento de la Junta Directiva.

El 11 de Diciembre de 2014 se aprobó el Acta Fundacional de la Asociación sin ánimo de lucro "Clúster de logística urbana de la Comunidad Valenciana". En el acta fundacional aparecen como miembros fundadores las siguientes entidades y empresas:

\footnotetext{
2 IVACE - Institut Valencià de Competitivitat Empresarial, es un Organismo Público de la Generalitat Valenciana (Gobierno Regional) y es uno de los partners del Proyecto Dorothy en la Región de Valencia.
} 
- Federación Valenciana de Empresarios del Transporte y la Logística. Se trata, como su nombre indica, de una Asociación Empresarial

- Instituto Tecnológico de Embalaje, Transporte y Logística. Es un ITENE es un Centro Tecnológico constituido como asociación privada con fines no lucrativos de ámbito nacional, fundada el 30 de mayo de 1994.

- Asociación para el Desarrollo de la Logística. Asociación privada que desrrolla formación y otras actividades en el campo de la logística.

- Empresa Movilidad Urbana Sostenible SL (MOVUS). Empresa privada que forma parte como partner del proyecto Dorothy. Actúa dentro del proyecto como CCP a través de su gerente Mauro Fiore

- IVACE (Instituto Valenciano de Competitividad Empresarial). Es una entidad de derecho público de la Generalitat Valenciana adscrita a la Consellería de Economía Sostenible, Sectores productivos, Comercio y Trabajo. Los fines principales del Instituto son la gestión de la política industrial de la Generalitat y el apoyo a las empresas en materia de innovación, emprendimiento, internacionalización y captación de inversiones.

- Asociación de Distribuidores a HORECA de la Comunidad Valenciana (ADISLEV). Asociación Empresarial sin ánimo de lucro que está integrada por gran parte de las empresas del sector de la distribución de bebidas y alimentación a HORECA radicadas en la Comunidad Valenciana

- Compañía de Almacenaje y Distribuciones Especiales SL (CADE). Empresa privada que forma parte como partner del proyecto Dorothy.

Como se observa, en la constitución aparecen tanto empresas privadas, como asociaciones empresariales y entidades públicas. Es decir,, se reúne a una parte importante de los agentes implicados en la logística urbana lo que permite garantizar la presencia de diferentes visiones e intereses que es uno de los objetivos del clúster.

Posteriormente se incorporaron al clúster distintos miembros hasta alcanzar la cifra actual de 17 miembros asociados.

Se nombró Presidente al D. Jaime Agramunt, que actúa en representación de la Federación Valenciana de Empresarios del Transporte y la Logística y Secretario a D. Javier Zabaleta Merí, que actúa en representación de ITENE.

El clúster solicitó, ante el Ministerio de Interior, la inscripción como Asociación sin ánimo de lucro el 19 de Diciembre de 2014. La resolución definitiva de inscripción de la Asociación se produjo en abril de 2015.

El objetivo principal del clúster, atendiendo a sus estatutos, será dar una respuesta estratégica conjunta al reto de lograr una logística y distribución urbana competitiva, 
eficiente y sostenible desde el punto de vista económico, social y medioambiental en el contexto actual y futuro de las poblaciones de la Comunitat Valenciana.

Para ello el clúster fomentará la cooperación empresarial, así como la cooperación público-privada para promover iniciativas innovadoras encaminadas a mejorar la competitividad de toda la cadena logística urbana y de las empresas que intervienen en la misma.

La Asociación se constituye asimismo con el objetivo general de aunar los distintos puntos de vista en la materia por parte de empresas (operadores, consultorías, proveedores de tecnología, etc.), centros de investigación (universidades, institutos, etc.) y la administración pública a nivel municipal y regional. El clúster busca dinamizar el sector de la logística urbana y, sobre todo, aportar a la misma nuevas soluciones, innovando y dando respuesta a un área básica para el desarrollo comercial y la calidad de vida de nuestras ciudades.

\section{4.- ACTUACIONES DEL CLUSTER DE LA COMUNIDAD VALENCIANA}

\section{1.- Dorothy Cluster Policy Event}

Los clústers de las cuatro regiones que participan en el proyecto Dorothy: Toscana, Lisboa y Valle del Tajo, Valencia y Oltenia, se reunieron en Valencia el 28 de Octubre de 2015 en un evento en el marco del cual se firmo un acuerdo de colaboración entre ellos.

El evento organizado en Valencia reunió a cerca de ciento treinta representantes de los gobiernos regionales y municipales, instituciones de la UE, empresas y organismos de investigación e innovación. Representó una gran oportunidad para discutir las perspectivas de futuro de la logística urbana y de las empresas e instituciones que operan en este sector en las regiones, a partir de las propuestas elaboradas por el Proyecto DOROTHY.

El consorcio europeo Dorothy ha definido un marco de áreas de actuación en las cuales cada región está trabajando (Plan de acción o Joint Action Plan- JAP). Entre ellas destacan algunos aspectos de relevante actualidad e importancia, como el impulso de los servicios de CargoBike, la innovación tecnológica sobre el control y gestión de plazas de carga y descarga, la coordinación de áreas de interés comercial donde se especifiquen planes ad hoc y la definición de Planes de Logística Urbana como documento estratégico de planificación y actuación urbana. Cada una de estas áreas representa una oportunidad para las empresas y asociaciones vinculadas al clúster para incrementar su potencial de mercado e innovar sus productos y tecnología. 


\section{2.- Participación como miembro de la Mesa de Movilidad del Ayuntamiento de Valencia}

El 24 de Noviembre de 2015, el Ayuntamiento de Valencia constituyó la Mesa de la Movilidad como "un órgano de participación y debate ciudadano, con carácter informativo y consultivo, para recoger las propuestas ciudadanas en un ámbito tan importante como el de la movilidad y con el objetivo de lograr un cambio de paradigma"

La Mesa de la Movilidad agrupa a cerca de 40 entidades ciudadanas, además de algunas empresas y las administraciones públicas relacionas con la movilidad.

"Transparencia, participación e inclusión" son las claves destacadas por el alcalde de Valencia, en el acto de constitución de la Mesa de Movilidad, "en el camino de esta ciudad hacia una movilidad sostenible, en la accesibilidad universal al servicio del transporte público y la recuperación social y ambiental del espacio público".

El clúster Logística Urbana de la Comunitat Valenciana, está presente desde su constitución en la Mesa de Movilidad del Ayuntamiento de Valencia participando en las diversas reuniones de la misma, en aquellos aspectos relacionados con la Logística Urbana.

\section{3.- Organización de un taller práctico, con el objetivo de fomentar la cooperación entre entidades en el ámbito de la logística urbana.}

El 11 de Febrero de 2016, en la sede social del clúster, ubicada en las instalaciones de ITENE, se llevó a cabo la organización de un taller de ideas relacionadas con la logística urbana.

Para el taller se solicitó a diversas empresas y entidades que aportasen ideas para la mejora de la logística urbana. Estas ideas se expusieron brevemente por parte de los promotores de las mismas y, tras esta exposición, se organizaron sesiones paralelas en las que se amplió información y se discutieron las mismas.

Las ideas presentadas y expuestas fueron las siguientes (entre paréntesis figura la empresa o entidad que presentó cada idea):

1. Reparto de mercancías en el área urbana de Valencia (A. T. Sarosa Levante, S.L.U)

2. E-cargo bike para la zona centro de Valencia (MOVUS)

3. Piloto/experiencia de carga y descarga con sensores de ocupación y reserva de espacios (MOVUS)

4. Proyecto piloto logística inversa para HORECA (MOVUS) 
5. Bases de partida para la organización de formacion en logistica urbana (UPV)

6. Multi-proveedor de bultos (RUMBO SISTEMAS)

7. Pilotos de validación de herramienta para la monitorización de consumos y eficiencia energética de flotas eléctricas (ITENE)

8. Marco cooperativo para la optimización de la distribucion de paqueteria (LISITT - Universidad de Valencia)

9. Última milla Valencia Centro (ENCICLE)

10. Micro plataformas de distribución. El internet de la logística (VANAPEDAL)

La fórmula utilizada de "taller de ideas" resultó de gran interés ya que animó la participación. En la misma jornada se informó sobre la actividad del clúster en la Mesa de Movilidad poniendo de manifiesto el interés que supone la presencia en la misma.

Igualmente se informó sobre los mecanismos existentes para la obtención de financiación para acciones de innovación y que podían ser utilizados en temas relacionados con la logística urbana.

Uno de los aspectos en los que se incidió fue el aspecto formativo. Se puso de manifiesto el interés de poner en marcha un "Curso de Gestores de Logística Urbana", de alrededor de 60 horas lectivas, encargando al partner Dorothy de la Universitat Politècnica de València (UPV) de explorar las posibilidades de financiación, la elaboración de una propuesta de programa y la posterior organización e impartición del mismo dentro del año 2016. La formación va destinada a: técnicos municipales, operadores logísticos, profesionales relacionados con el ámbito de la logística urbana y otros técnicos interesados.

En este sentido, se participó en una reunión celebrada en la UPV el 8 de marzo de 2016 y convocada por el proyecto Climate Kic Spain, en la que se planteó la realización del Curso de Gestores de Movilidad con financiación de Climate Kic. Se ha enviado una propuesta detallada a Climate Kic y se está a la espera de respuesta.

\section{5.- RESUMEN Y PRINCIPALES CONCLUSIONES}

El Proyecto Dorothy "Development Of RegiOnal clusTers for researcH and implementation environmental friendlY urban logistics" (www.clusterdorothy.com), forma parte del 7th Framework Programme of the EU que finaliza el 30 de Junio de 2016 plantea como uno de sus objetivos principales la creación de clusters, a nivel regional que aborden temas relacionados con la logística urbana con participación de los agentes implicados. 
La creación de un clúster en logística urbana, y su puesta en marcha, sin disponer de fondos económicos para su dinamización inicial, es un proceso complejo que requiere abordar diversos pasos con continuidad y llevar a cabo distintas gestiones. Al menos este ha sido el caso de la creación del clúster de la Región de Valencia.

El presente esquema resume las acciones que se han llevado a cabo para llegar a crear la Asociación sin ánimo de lucro "Clúster Logística Urbana de la Comunitat Valenciana" que es hoy una realidad derivada del proyecto Dorothy.

Fecha

$01-$ jul-13

Primeros
meses

22-may-14
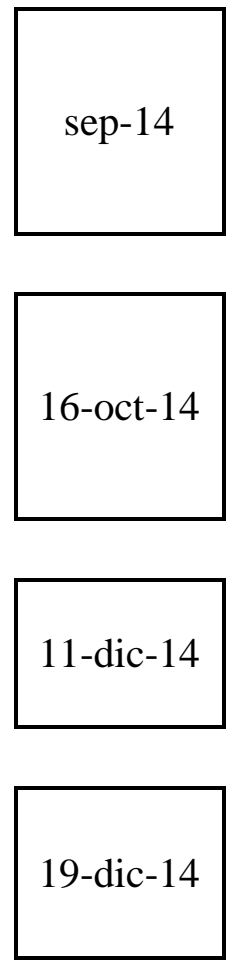

abr-15

\section{Acción}

Inicio Proyecto Dorothy

Estudio de la situación de la logística urbana en la Región de Valencia

Reunión en la Cámara de Comercio: "El nuevo clúster de lo logística urbana de la Comunidad Valenciana" presentando el proyecto Dorothy

Difusión de la propuesta de clúster en la Jornada Infoday Proebike con el título

"Dorothy: El nuevo Cluster de la logística urbana de la Comunidad Valenciana"

Reunión en IVACE de posibles interesados en el clúster. Los interesados debían manifestar por escrito su interés. Se fijó fecha para la reunión fundacional

Reunión para "Acta Fundacional" con aprobación de estatutos y nombramiento de Junta Directiva

Presentación de inscripción de la Asociación sin ánimo de lucro "Clúster Logística Urbana de la Comunitat Valenciana"

Aprobación de la inscripción por parte del Ministerio de Interior 
Tras la creación del clúster, y en el corto período de tiempo transcurrido, la actividad del mismo ha sido notable. esta actividad se resume en el siguiente esquema:

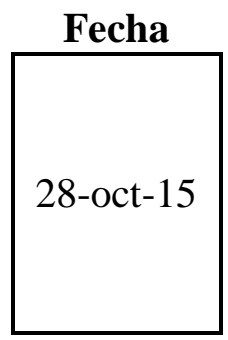

Acción

Dorothy Cluster Policy Event. Reunión de los clústers de las regiones del proyecto Dorothy: Toscana, Lisboa y Valle del Tajo, Valencia y Oltenia. Firma de acuerdo de colaboración entre todos ellos

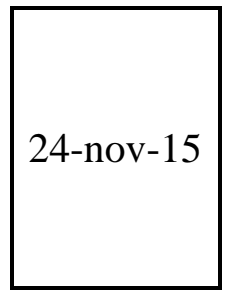

Constitución de la Mesa de la Movilidad del Ayuntamiento de Valencia de la que forma parte el "Clúster Logística Urbana de la Comunitat Valenciana". Posterior presencia en las reuniones de la Mesa

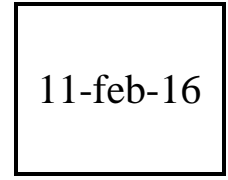

Organización de un taller práctico, con el objetivo de fomentar la cooperación entre entidades en el ámbito de la logística urbana

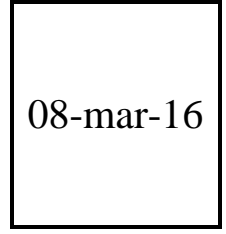

Participación en reunión de Climate Kic Spain. Propuesta detallada de Curso de Gestores de Logística Urbana con solicitud de financiación a través de Climate Kic.

Como conclusión final cabe señalar que se ha dado cumplimiento a unos de lso objetivos principales del Proyecto Dorothy a través de la creación y puesta en marcha del "Clúster Logística Urbana de la Comunitat Valenciana". 